\title{
Soll die Betriebswirtschaftslehre die Interessen anderer Akteure explizit berücksichtigen?
}

Ute Schmiel

Lehrstuhl für Unternehmensbesteuerung, Fakultät für Wirtschaftswissenschaften, Universität Duisburg-Essen, Essen, Germany

Received November 30 2017; Accepted July 122018

Zusammenfassung: In der BWL wird die Auffassung vertreten, dass die BWL bei unternehmenspolitischen Ziel-Mittel-Analysen die Interessen anderer Akteure nicht explizit berücksichtigen muss. Diese Auffassung wird implizit oder explizit mit der Harmonie zwischen Unternehmenszielen und Interessen anderer Akteure begründet. Dieser Beitrag wendet jedoch aus einer kritisch-rationalen Perspektive ein, dass diese Harmoniehypothesen nicht empirisch bewährt sind. Deshalb können Akteure ihre eigenen Interessen nicht uneingeschränkt verfolgen. Vielmehr sollte der gesellschaftliche Wert von Märkten darin bestehen, dass Akteure im Eigeninteresse handeln können und dabei legitime Interessen anderer Akteure wahren sollen. Der Beitrag zeigt außerdem auf, wie die BWL Ziel-Mittel-Analysen durchführen sollte, die sich an einem solchen Wert von Märkten orientieren.

Schlagwörter: Gewinnerzielung • kritischer Rationalismus • legitime Interessen • Ziel-Mittel-Analyse • Verfolgung eigener Interessen

Abstract: Business administration argues that means-end-analysis in the interest of firms does not need to take the interests of other actors into account. Its implicit or explicit reason is that there is a harmony between firm goals and the interests of other actors. This study objects from a critical rationalist perspective that such harmony hypotheses are not empirically confirmed. Because of this, actors are not truly free to pursue their own interests. Instead, this study argues that actors on markets are allowed to pursue their own interests as long as they consider the legitimate interests of other actors at the same time. The study goes on to show how business administration should analyze means-end-statements that try to realize this market value.

Keywords: Critical rationalism • legitimate interests • means-end-analysis • profit orientation • pursuing own interests

\section{Einleitung}

Die Betriebswirtschaftslehre (BWL) wird häufig als „anwendungsorientierte Wissenschaft" verstanden (siehe explizit Köhler et. al. 2007, S. 138; Schanz 2009, S. 81-88; Wöhe 2016, S. 4 f.). Das heißt, dass die wissenschaftliche Aufgabe der BWL nicht auf explikative Aussagen, also auf das Erkennen und Formulieren von Zusammenhängen, beschränkt wird. Explikative Aussagen wären beispielsweise Aussagen über die Funktionsweise von Märkten oder über das Handeln von Unternehmen. Vertreterlnnen einer anwendungsorientierten BWL sehen es vielmehr auch als wissenschaftliche Aufgabe der BWL an, mithilfe von Ziel-Mittel-Analysen Gestaltungsvorschläge zu unterbreiten. Solche Gestaltungsvorschläge können unterschiedliche Adressaten haben. Zum einen können sie vorrangig die Wirtschaftsordnung in den Blick nehmen. Letzteres ist der Fall, wenn gesetzliche oder außergesetzliche Regeln zur Wirtschaftsordnung überprüft oder Vorschläge zu gesetzlichen oder außergesetzlichen Regeln zur Wirtschaftsordnung unterbreitet werden. 
Zum anderen können sich Gestaltungsaussagen primär an Individuen oder Unternehmen richten. Ein Beispiel für eine solche Ziel-Mittel-Aussage wäre Wenn Unternehmen den Shareholder Value maximieren wollen, dann ist es bei Risikoneutralität ein geeignetes Mittel, die Investition mit dem höchsten Erwartungswert des Kapitalwertes zu wählen (Wöhe 2016, S. 505-507) oder Wenn Unternehmen Gewinne maximieren wollen, dann ist legale aggressive Steuergestaltung ${ }^{1}$ ein geeignetes Mittel, sofern die Risiken abschätzbar und kein Reputationsschaden zu erwarten ist (Grotherr 2015).

Der vorliegende Beitrag fokussiert auf unternehmenspolitische Ziel-Mittel-Analysen, beispielsweise auf Ziel-Mittel-Analysen im Sinne der gerade genannten Investitions- oder Finanzierungsentscheidungen oder auch im Hinblick auf Produktions-, Organisations- oder Personalentscheidungen. Der vorliegende Beitrag fragt, ob sich die BWL bei solchen unternehmenspolitischen Ziel-Mittel-Analysen auf die Vorteilhaftigkeit aus Sicht des Unternehmens oder (was durchaus unterschiedlich sein kann) aus Sicht der Anteilseigner beschränken kann oder ob sie explizit auch Interessen anderer Akteure berücksichtigen soll.

Die Frage nach der Berücksichtigung von Interessen anderer Akteure in unternehmenspolitischen Ziel-MittelAnalysen ist in unterschiedlichen Kontexten national und international diskutiert worden. Dabei findet die Diskussion teilweise auf methodologischer Ebene (was sollte die BWL tun?) und teilweise auf Objektebene (was sollten Unternehmen tun?) statt. Eine methodologische Diskussion wird im deutschsprachigen Raum im Zusammenhang mit dem Gegenstand der BWL (Köhler et. al. 2007, S. 138; Schweitzer 2009, S. 9-6; Wöhe 2016, S. 3-13) geführt. Eine Diskussion auf Objektebene finden wir beispielsweise im Zusammenhang mit verantwortlichem Handeln als Business Case (Carroll und Shabana 2010; kritisch Thielemann 2008, S. 202208), der Zulässigkeit des Shareholder-Value-Ansatzes (Sundaram und Inkpen 2004) oder in Analysen des Kapitalismus allgemein (Gaus 2010, S. 93 f.). In jüngerer Zeit wird diese Frage auch im Kontext aggressiver Steuergestaltung geführt, wenn etwa analysiert wird, ob der Vorstand einer AG gegenüber den Aktionären zu aggressiver Steuergestaltung nicht sogar verpflichtet

1 Aggressive Steuergestaltungen sind nach Grotherr (2015) dadurch gekennzeichnet, dass sie sich auf Einkünfte aus international mobilen Aktivitäten beziehen, das tatsächliche Motiv darin besteht, einen signifikanten Steuervorteil zu erlangen, die Gesamtsteuerbelastung für betreffende Gestaltungen im Vergleich zur in den tangierten Staaten grundsätzlich zu erwartenden Unternehmenssteuerbelastung erheblich niedriger ist, die gewählte Gestaltung ohne nennenswerte wirtschaftliche Substanz ist und in der Summe zulasten des Steueraufkommens aller tangierten Fiski geht. ist (Grotherr 2015) oder wenn ausgeführt wird, dass es nicht sinnvoll sei, „gegenüber Steuerpflichtigen ihre Neigung zur Steuervermeidung anzuprangern, die zu den festen Merkmalen einer privatwirtschaftlich organisierten Ökonomie gehört" (Wagner 2014, S. 1136).

Der vorliegende Beitrag unterscheidet sich von diesen Diskussionen in verschiedener Hinsicht. Von den Diskussionen auf Objektebene zunächst darin, dass in diesem Beitrag eine methodologische Perspektive eingenommen wird. Es geht also um die Frage, ob die BWL Interessen anderer Akteure in unternehmenspolitischen Ziel-Mittel-Aussagen explizit berücksichtigen soll. Das schließt nicht aus, Argumente, die dafür oder dagegen sprechen, dass Unternehmen Interessen anderer Akteure explizit in Entscheidungen berücksichtigen, auch für die Beantwortung der hier thematisierten Frage zu verwenden. Von den bisher vorliegenden methodologischen Erörterungen grenzt sich der Beitrag zum einen dadurch ab, dass er explizit eine kritisch-rationale Perspektive im Sinne von Albert $(1985,1999)$ und Gadenne (2006) einnimmt. Der Beitrag fragt, welche Anforderungen danach Ziel-Mittel-Analysen erfüllen sollen, und was daraus für die Berücksichtigung von Interessen anderer Akteure in unternehmenspolitischen Ziel-MittelAnalysen folgt. Zum anderen gelangt dieser Beitrag im Unterschied zu vorhandenen methodologischen Diskussionen zu dem Ergebnis, dass eine Begründung dafür fehlt, die Interessen anderer Akteure in unternehmenspolitischen Ziel-Mittel-Aussagen nicht explizit zu berücksichtigen. Denn diese Nichtberücksichtigung, so lautet die Argumentation, baut auf der Hypothese auf, dass individuelle Vorteilsverfolgung gesamtwirtschaftlich vorteilhaft ist und damit automatisch die Interessen anderer Akteure in den Blick nimmt. Diese Hypothese ist aber empirisch nicht bewährt (und damit im kritischrationalen Sinne nicht adäquat). Der Beitrag bleibt bei diesem Ergebnis (das zum Teil auch in der Diskussion auf der Objektebene zu finden ist) nicht stehen. Er untersucht vielmehr, wie die BWL die Interessen anderer Akteure in unternehmenspolitischen Ziel-MittelAussagen berücksichtigen sollte. Dazu ist er folgendermaßen aufgebaut: Kapitel 2 belegt zunächst mit einigen Zitaten, dass die Auffassung vertreten wird, die BWL bräuchte in unternehmenspolitischen Analysen die Interessen anderer Akteure nicht explizit zu berücksichtigen. Kapitel 3 legt die hier vorgelegte kritisch-rationale Position dar. Kapitel 4 zeigt auf, dass aus kritisch-rationaler Sicht keine Argumente dafür vorliegen, die Interessen anderer Akteure in unternehmenspolitischen Ziel-MittelAnalysen nicht explizit zu berücksichtigen. Weil vielmehr in unternehmenspolitischen Ziel-Mittel-Analysen die Interessen anderer Akteure explizit berücksichtigt werden sollten, stellt sich die Frage, wie dies erfolgen 
sollte. Diese Frage diskutiert Kapitel 5. Kapitel 6 fasst die Ergebnisse zusammen.

\section{Wird tatsächlich die Auffassung vertreten, unternehmenspolitische Ziel- Mittel-Analysen bräuchten die Interessen anderer Akteure nicht explizit zu berücksichtigen?}

Dieser Beitrag untersucht auf einer methodologischen Ebene (was sollte die BWL tun?), ob in unternehmenspolitischen Ziel-Mittel-Analysen die Interessen anderer Akteure berücksichtigt werden sollten. Kapitel 2 soll exemplarisch aufzeigen, dass in der betriebswirtschaftlichen Standardliteratur auf einer methodologischen Ebene die Auffassung vertreten wird, die BWL bräuchte in unternehmenspolitischen Ziel-Mittel-Analysen die Interessen anderer Akteure nicht explizit zu berücksichtigen. Methodologische Ausführungen sind vor allem in Lehrbüchern enthalten. Im Folgenden werden mit „Wöhe“ und „Bea und Schweitzer“ zwei Standardlehrbücher der BWL sowie mit einem Artikel aus dem Handwörterbuch der BWL ein Überblicksaufsatz zum state of the art zugrunde gelegt.

Nach der von Bea und Schweitzer vorgelegten „Allgemeinen Betriebswirtschaftslehre“ ist das Identitätsprinzip der BWL das Ergiebigkeitsprinzip. Dieses Prinzip wird so interpretiert, „dass mit den vorhandenen knappen Mitteln (Gütern) optimale Ausprägungen der gesetzten Ziele erreicht werden“ (Schweitzer 2009, S. 54). Zwar wird betont, dass das Ergiebigkeitsprinzip alle denkbaren Ziele einbezieht (Schweitzer 2009, S. 55). Gleichwohl wird ausgeführt (und nicht weiter kritisiert), dass private Unternehmen die ökonomische Ergiebigkeit als dominierendes Ziel verfolgen können. Hierzu zählen beispielsweise Umsatz, Erfolg, Umsatz-, Gesamtkapital- oder Eigenkapitalrentabilität (Schweitzer 2009, S. 58 f.).

Eine ähnliche Auffassung wird in dem Handwörterbuch der BWL vertreten. Dort heißt es zum Erkenntnisgegenstand der BWL: „Allgemeiner lässt sich der Erkenntnisgegenstand der BWL charakterisieren, wenn man von der Knappheit der verfügbaren Ressourcen ausgeht, die grundsätzlich das Wirtschaften in betrieblichen Institutionen bestimmt, und daran anknüpfend die Frage nach der Effizienz der Ziel-Mittel-Verhältnisse aufwirft. Dabei werden nicht von vornherein bestimmte Zielinhalte postuliert, sondern diese ergeben sich aus der empirischen Feststellung betrieblicher Zielsysteme ... Die verbindende Klammer der vielfältigen
Untersuchungsansätze ... ist die jeweilige Berücksichtigung von Input-Output-Beziehungen, die unter den Stichwörtern Effizienz, Ergiebigkeit oder Wirtschaftlichkeit behandelt werden." (Köhler et al. 2007, S. 138, im Original teilweise hervorgehoben).

Während die von Bea und Schweitzer vorgelegte „Allgemeine Betriebswirtschaftslehre" sowie das Handwörterbuch der BWL damit die Auffassung vertreten, dass die BWL die Interessen anderer Akteure nicht explizit zu berücksichtigen braucht, geht die von Wöhe begründete „Allgemeine Betriebswirtschaftslehre" darüber hinaus. Wöhe führt hinsichtlich des Erkenntnisgegenstands der BWL aus: "Die wirtschaftstheoretisch fundierte Betriebswirtschaftslehre begnügt sich mit einer sehr engen Fachabgrenzung. Bei der Untersuchung betrieblichen Geschehens beschränkt sie sich auf das Wirtschaften im Betrieb und gelangt so zu ihrem (engen) Erkenntnisobjekt. Das Auswahlprinzip zur Beurteilung betrieblicher Handlungsalternativen ist das ökonomische Prinzip ... Das ökonomische Prinzip verlangt, knappe Mittel (Input) so einzusetzen, dass ein größtmöglicher Output an Gütern (Sachleistungen) und Dienstleistungen erzielt wird. Die Effizienz, d. h. das Verhältnis von wertmäßigem Output zu wertmäßigem Input, ist für Ökonomen der allein gültige Maßstab zur Beurteilung betrieblicher Handlungen." (Wöhe 2016, S. 8, im Original hervorgehoben). Nach Wöhe braucht also die BWL Interessen anderer Akteure nicht nur nicht explizit zu berücksichtigen. Nimmt man diese Ausführungen beim Wort, dürfte sie diese nicht explizit berücksichtigen.

Der hier vorgelegte Beitrag vertritt eine entgegengesetzte Auffassung: Weil es keine Gründe dafür gibt, Interessen anderer Akteure in unternehmenspolitischen Ziel-Mittel-Analysen nicht zu berücksichtigen, ist es auch nicht begründet, den Erkenntnisgegenstandes der BWL auf (ökonomische) Effizienz zu beschränken. Weil dieser Beitrag die Anforderungen an Ziel-Mittel-Aussagen aus kritisch-rationaler Sicht untersucht, werden im nächsten Kapitel zunächst die kritisch-rationalen Anforderungen an Ziel-Mittel-Analysen erörtert.

\section{Kritisch-rationale Anforderungen an Ziel-Mittel-Analysen}

Dieser Beitrag erörtert auf einer methodologischen Ebene die Frage, ob die BWL in unternehmenspolitischen Ziel-Mittel-Analysen die Interessen anderer Akteure explizit berücksichtigen sollte und nimmt dabei eine kritisch-rationale Perspektive ein (Heitfeldt und Schmiel 2017, S. 99-101). Grundlegende Idee eines kritisch-rationalen Wissenschaftsverständnisses 
ist, Annahmen und Prinzipien herauszuarbeiten und sie so der Kritik zugänglich zu machen oder wie Gadenne (2006, S. 107) ausführt „[to] work out the philosophical assumptions and principles that lie behind our actions and lives, so that they can be criticised and perhaps improved“ (Gadenne 2006, S. 107).

Kritisch-rationale Methodologie zeichnet sich erstens dadurch aus, dass sie eine Erkenntnis der Wirklichkeit für möglich hält. Diese steht aber unter dem Vorbehalt der Subjektivität der Wahrnehmung, sie ist theoriebeladen (Popper 1968, S. 59, Fußnote 1). Kritisch-rationale Methodologie geht also von einem kritischen Realismus aus. Zweitens lehnt eine kritisch-rationale Methodologie absolut sichere Erkenntnis ebenso ab (konsequenter Fallibilismus) wie die Möglichkeit, Werte abschließend begründen zu können. Deshalb legt sie drittens einen methodologischen Revisionismus zugrunde, der die kritische Prüfung wissenschaftlicher Ergebnisse und das kritische Hinterfragen von Begründungen von Werten betont (Albert 1999, S. 9-20). In diesem Beitrag wird eine Methodologie zugrunde gelegt, die sich an dem Verständnis des kritischen Rationalismus von Albert und Gadenne orientiert. Aus Sicht dieses Beitrags ist vor allem das Folgende wichtig: Albert und Gadenne verstehen methodologische Regeln als Heuristiken zur Erreichung von Wissenschaftszielen (Albert 1985; 1999; Gadenne 2006). Aus der Perspektive des kritischen Rationalismus weist jede Erkenntnis einen vorläufigen Charakter auf und dieser vorläufige Charakter bezieht sich ebenso auf den kritischen Rationalismus selbst. Ein kritisch-rationales Wissenschaftsverständnis würde seinen eigenen Anforderungen nicht genügen, wenn es seine methodologischen Regeln dogmatisieren würde. Ebenso wenig wie andere Ziele und Mittel kann ein kritisch-rationales Wissenschaftsverständnis letztbegründet werden. Es können lediglich Argumente dafür angeführt werden, warum kritisch-rationale Methodologie eine geeignete Technologie ist, um das Wissenschaftsziel zu erreichen (Gadenne 2006, S. 99) und diese kritisch-rationale Methodologie sowie diese Argumente zur kritischen Diskussion zu stellen. In diesem Sinne werden hier kritisch-rationale Anforderungen an unternehmenspolitische Ziel-Mittel-Aussagen verstanden. Des Weiteren wendet Albert kritischen Rationalismus auch auf die Gestaltung der Gesellschaftsordnung und allgemein auf Gestaltungsaussagen an (Albert 1985, S. 218-229; Albert 1999, S. 151-165) und diese methodologischen Regeln für Gestaltungsaussagen sind für die hier erörterten unternehmenspolitischen Ziel-Mittel-Analysen relevant. Außerdem nimmt Gadenne zum kritisch-rationalen Theorieverständnis Stellung (Gadenne 2006). Auch dies ist für die folgende Analyse wichtig, weil Ziel-Mittel-Aussagen solche theoretischen Aussagen zugrunde liegen sollten, die aus Sicht des kritischen Rationalismus adäquat sind.

Aus kritisch-rationaler Sicht hat die Analyse von Ziel-Mittel-Aussagen eine ethische und eine erfahrungswissenschaftliche Dimension (Opp 2014, S. 249-269). Im Rahmen der ethischen Dimension ist zunächst das Postulat der Werturteilsfreiheit zu beachten. Weil Werte nicht letztbegründet werden können, sind Ziele als alternative Vorschläge zu anderen Zielen zu verstehen. Sie sind außerdem durch Rückgriff auf allgemeinere Ziele oder Werte ethisch zu reflektieren. Ferner sind sie zur kritischen Diskussion zu stellen, d. h. sie dürfen nicht als nicht hinterfragbare „letzte Werte“ dogmatisiert werden (Albert 1985, S. 80-87, 94-101, 218-229; Albert 1999, S. 65-76; Vanberg 2014, S. 344-348). Deshalb ergibt sich für die in der Einleitung angeführten Beispiele für Ziel-Mittel-Aussagen

- Wenn Unternehmen den Shareholder Value maximieren wollen, dann ist es bei Risikoneutralität ein geeignetes Mittel, die Investition mit dem höchsten Erwartungswert des Kapitalwertes zu wählen und

- Wenn Unternehmen Gewinne maximieren wollen, dann ist legale aggressive Steuergestaltung ein geeignetes Mittel, sofern die Risiken abschätzbar und kein Reputationsschaden zu erwarten ist.

die Notwendigkeit, zu fragen, ob Maximierung des Shareholder Value bzw. Gewinnmaximierung ohne explizite Berücksichtigung von Interessen anderer Akteure mit allgemeineren Zielen oder Werten vereinbar ist. Selbst wenn dies der Fall wäre (dieser Beitrag argumentiert dagegen), widerspräche es gleichwohl kritisch-rationaler Methodologie, Gewinnmaximierung als alternativloses letztes Ziel zu verstehen. Gewinnmaximierung ohne explizite Berücksichtigung der Interessen anderer Akteure wäre dann vielmehr allenfalls ein vorläufig begründetes Ziel.

Die erfahrungswissenschaftliche Dimension der Ziel-Mittel-Analyse resultiert aus dem Prinzip „Sollen impliziert Können“ (Albert 1985, S. 98). Danach reicht es nicht aus, dass gute Gründe dafür sprechen, dass Unternehmen bestimmte Ziele verfolgen. Vielmehr ist es ebenso notwendig, dass diese Ziele umgesetzt werden können (Realisierbarkeitspostulat). Diese Anforderung gilt auch für die allgemeineren Ziele oder Werte, auf die Unternehmensziele zurückgeführt werden. Auch diese sollen realisierbar sein. Realisierbarkeit meint, dass die Bedingungen, die gemäß erfahrungswissenschaftlicher Hypothesen für die Zielerreichung notwendig sind, auch geschaffen werden können. Ist dies nicht der Fall, weil es sich um kontrafaktische Bedingungen handelt, gibt es keinen Grund, davon auszugehen, dass 
Ziele erreicht werden können. Ein Nirvana-Approach, also das Zugrundelegen eines nicht realisierbaren Ideals wird aus kritisch-rationaler Perspektive abgelehnt (Albert, 1985, S. 218-229). Eine kritisch-rationale Methodologie stellt außerdem Anforderungen an diese erfahrungswissenschaftlichen Hypothesen. Neben weiteren Adäquanzbedingungen ist es erforderlich, dass Antezedensbedingungen und Hypothesen vorläufig empirisch bestätigt sind (Opp 2014, S. 205-213). Hierzu zählen solche Hypothesen, die bisher kritischen Tests am besten standgehalten haben (Gadenne, 2006, S. 106). Weil aber nach kritisch-rationalem Verständnis aufgrund des Induktionsproblems keine Verifikation und aufgrund der Theoriebeladenheit der Beobachtung keine endgültige Falsifikation möglich ist, können Hypothesen nur vorläufig empirisch bewährt sein (Gadenne 2006).

Eine kritisch-rational adäquate Ziel-Mittel-Analyse erfordert schließlich, die Mittel, mit denen die zugrunde gelegten Ziele erreicht werden sollen, aus ethischer und erfahrungswissenschaftlicher Perspektive kritisch zu reflektieren. Insbesondere sind sie dahingehend zu untersuchen, ob sie geeignet sind, Ziele zu erreichen oder ob geeignetere Mittel zur Zielerreichung zur Verfügung stehen. Des Weiteren ist zu untersuchen, ob sie aufgrund ihrer Nebenwirkungen mit anderen Zielen oder Mitteln in Konflikt treten (Albert 1985, S. 80-87, 218229). Abbildung 1 fasst diese kritisch-rationalen Anforderungen an Ziel-Mittel-Aussagen zusammen.

Die in diesem Beitrag beispielhaft angeführten unternehmenspolitischen Ziel-Mittel-Aussagen Wenn Unternehmen den Shareholder Value maximieren wollen, dann ist es bei Risikoneutralität ein geeignetes Mittel, die Investition mit dem höchsten Erwartungswert des Kapitalwertes zu wählen und wenn Unternehmen Gewinne maximieren wollen, dann ist legale aggressive Steuergestaltung ein geeignetes Mittel, sofern die Risiken abschätzbar und kein Reputationsschaden zu erwarten ist beschränken sich auf die Vorteilhaftigkeit aus Sicht des Unternehmens oder dessen Anteilseigner ohne explizite Berücksichtigung der Interessen anderer Akteure. Dies wäre zulässig, wenn das zugrunde gelegte Ziel „Gewinnmaximierung“ seinerseits mit allgemeineren Zielen oder Prinzipien vereinbar wäre. Diese allgemeineren Ziele müssten selbst wiederum ethisch reflektiert und erfahrungswissenschaftlich auf ihre Realisierbarkeit hin untersucht werden.

Wenn also in unternehmenspolitischen Aussagen ausschließlich die Vorteilhaftigkeit aus Unternehmenssicht ohne explizite Berücksichtigung der Interessen anderer Akteure zugrunde gelegt wird, bedarf es hierfür Argumente und diese - so wird in Kapitel 4 deutlich werden - liegen aus kritisch-rationaler Sicht nicht vor.

\section{Berücksichtigen unternehmenspolitische Ziel- Mittel-Aussagen die Interessen anderer Akteure automatisch?}

Gegenstand dieses Beitrags ist die Frage, ob die BWL in unternehmenspolitischen Ziel-Mittel-Analysen die Interessen anderer Akteure berücksichtigen sollte. Wenn die Auffassung vertreten wird, dass die BWL in unternehmenspolitischen Ziel-Mittel-Analysen die Interessen anderer Akteure nicht explizit in den Blick zu nehmen braucht, wird dies implizit oder explizit mit der

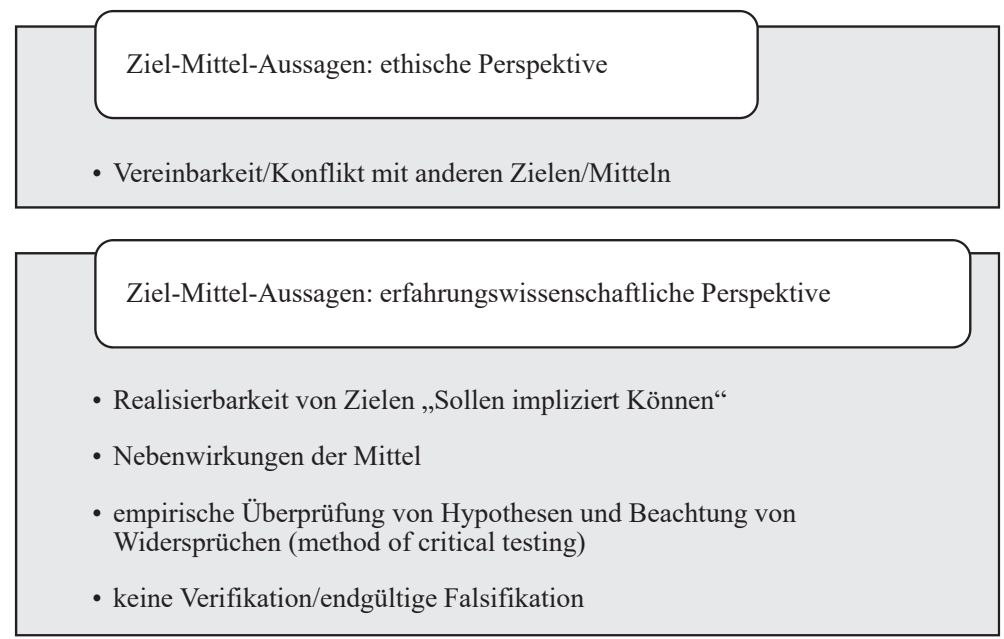

Abbildung 1. Kritisch-rationale Anforderungen an Ziel-Mittel-Aussagen 
Harmonie zwischen Unternehmenszielen und Interessen anderer Akteure begründet. Danach berücksichtigt das Verfolgen individueller Vorteilhaftigkeit automatisch die Interessen anderer Akteure, sodass deren explizite Beachtung nicht erforderlich ist. Auch diese Hypothese wurde in unterschiedlichen Kontexten (insbesondere auf Objektebene) angeführt. Im Kern geht diese Überlegung, dass Wirtschaftssubjekte aus Eigeninteresse die Interessen anderer in den Blick nehmen, auf Adam Smith zurück (Smith 1966, S. 17; dazu Weise et al. 2005, S. 409). Sie wird in der neoklassischen Wohlfahrtsökonomie auf die Harmonie individueller Nutzenmaximierung und Pareto-Effizienz auf Märkten in einem allgemeinen Konkurrenzgleichgewicht verengt. Nach der neoklassischen Markttheorie sind Märkte unter bestimmten Voraussetzungen in einem allgemeinen Konkurrenzgleichgewicht. Die Markthypothese lautet: Wenn alle Güter Märkte haben, alle Marktteilnehmer Preisnehmer und reine Mengenanpasser sind, sich rational verhalten (was mit nutzenmaximierendem Handeln gleichgesetzt wird), über vollkommene Information verfügen, die Anpassung an veränderte Marktbedingungen keine Zeit erfordert und kostenlos ist, Faktorbestände, Wissen und Präferenzen gegeben sind, ..., dann liegt ein Marktgleichgewicht vor (siehe zu diesen und weiteren Voraussetzungen Kleinewefers 2008, S. 116). Ein Markt im vollkommenen Konkurrenzgleichgewicht ist durch gesamtwirtschaftliche Pareto-Effizienz gekennzeichnet (Weise et al. 2005, S. 405 f.; Kleinewefers 2008, S. 42-44, S. 103 f.; Mas-Colell et al., 1995, S. 311-316). Die individuelle Nutzenmaximierung zeigt an, dass aus individueller Sicht Ressourcen bestmöglich verwendet wurden. Der gesamtwirtschaftliche Nutzen indiziert, dass dies auch aus gesamtwirtschaftlicher
Sicht gilt. Der gesamtwirtschaftliche Nutzen ist nach diesem Verständnis identisch mit der Summe individueller Nutzen (kritisch Etzioni 2015). Individuelle und gesamtwirtschaftliche Interessen stimmen automatisch überein. Dabei sind weder der individuelle Nutzen noch der gesamtwirtschaftliche Nutzen inhaltlich spezifiziert. Die folgende Abbildung 2 zeigt diesen Zusammenhang auf, indem sie die neoklassische Markthypothese in eine Ziel-Mittel-Analyse integriert.

Nach hier vertretener Auffassung ist diese Harmoniehypothese in dieser neoklassischen Formulierung problematisch. Neben der (hier nicht weiter erörterten) ethischen Kritik an dem Ziel der Pareto-Effizienz (Ulrich 2008, S. 203-207) sprechen gegen eine solche Harmoniehypothese erfahrungswissenschaftliche Argumente. Denn die Antezedensbedingungen vollkommener Märkte (Wenn alle Güter Märkte haben, alle Marktteilnehmer Preisnehmer und reine Mengenanpasser sind, sich rational verhalten (was mit nutzenmaximierendem Handeln gleichgesetzt wird), über vollkommene Information verfügen, die Anpassung an veränderte Marktbedingungen keine Zeit erfordert und kostenlos ist, Faktorbestände, Wissen und Präferenzen gegeben sind) sind nicht realisiert und auch nicht realisierbar (Nutzinger 1992, S. 47-51). Beispielsweise setzt ein vollkommener Markt Sicherheit oder stochastische Unsicherheit, d. h. den Fall voraus, dass Wirtschaftssubjekte sämtliche Zukunftslagen und deren Eintrittswahrscheinlichkeiten kennen. Nur unter dieser Prämisse können Wirtschaftssubjekte im Sinne der o.g. Markthypothese objektiv-rational handeln (Vanberg 2002, S. 11-14; 2004, S. 8, 17) und außerdem haben sie nur unter dieser Prämisse vollkommene Information. Geht man hingegen von genuiner Unsicherheit, also davon aus, dass Umweltzustände

Marktziel (Wert): Pareto-Effizienz

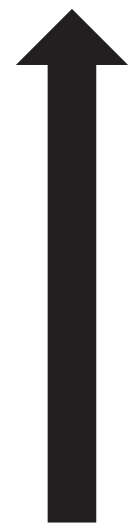

Zielerreichung durch Mittel: neoklassische Markttheorie

Mittel: Marktbedingungen eines Marktgleichgewichts bei vollkommener Konkurrenz

Abbildung 2. Harmoniehypothese in neoklassischer Formulierung als Teil einer Ziel-Mittel-Analyse 
eintreten können, die Wirtschaftssubjekten beim Treffen ihrer Entscheidung nicht bekannt sind (Shackle 1972, S. 3 f.; Beckert 1996, S. 814 f.; Schneider 2001, S. 370-378, 469), ist neoklassische Theorie auf Märkte nicht anwendbar (Witt 2009, S. 366-369; Vanberg, 2001). Dies lässt sich neben anderem daran verdeutlichen, dass unter genuiner Unsicherheit objektiv-rationales Handeln nicht definiert ist. Wirtschaftssubjekte können nur subjektiv-rational handeln, d. h. vor dem Hintergrund ihres subjektiven Wissens ihre Ziele verfolgen (Beckert 1996, S. 817-823; Vanberg 2002, S. 10-15). Damit erfüllt die neoklassisch gedeutete Harmoniehypothese die kritisch-rationale Anforderung der Realisierbarkeit von Umweltbedingungen nicht.

Des Weiteren spricht gegen die neoklassisch verstandene Harmoniehypothese, dass die neoklassische Markttheorie für zahlreiche Probleme der realen Welt blind ist, weil diese in vollkommenen Märkten unter Sicherheit oder stochastischer Unsicherheit nicht definiert sind. Hierzu zählen beispielsweise absolute Ressourcenknappheit (Holstein 2003, S. 38-68, 288), Innovationen und das Lernen von Individuen, Illiquidität, der Gebrauch von Geld oder das Vorhandensein von Institutionen wie beispielsweise Unternehmen (Shubik, 2007). Deshalb verknüpfen institutionenökonomische Theorien neoklassische Theorie häufig mit (partiell) asymmetrischer Information oder positiven Transaktionskosten. Werden Agency-Kosten oder Transaktionskosten minimiert, so ist dann die Überlegung, wird zumindest näherungsweise Harmonie zwischen Unternehmenszielen und gesamtwirtschaftlichen Zielen erreicht. Solche Hybridtheorien verknüpfen dann allerdings sich widersprechende Annahmen.

Beispielsweise sind Eigenkapitalgeber in der AgencyTheory im Hinblick auf einige Sachverhalte perfekt informiert und im Hinblick auf einige Sachverhalte haben sie unvollständige Information (Richter und Furubotn 2005, S. 508-533). Aus widersprüchlichen Aussagen kann aber jede beliebige Aussage gefolgert werden (Albert 1985, S. 56 f.). Aus kritisch-rationaler Sicht sind solche Hybridtheorien deshalb ebenfalls nicht adäquat und damit ebenfalls nicht geeignet, die Harmoniehypothese zu stützen.

Neben dem Bezug auf die neoklassische Markttheorie wird Ziel-Mittel-Analysen explizit oder implizit eine weitergefasste Harmoniehypothese zugrunde gelegt. Eine weitergefasste Harmoniehypothese vertritt explizit Wöhe, der in der „Allgemeinen Betriebswirtschaftslehre“ darlegt: „Die von Adam Smith entdeckte unsichtbare Hand des marktwirtschaftlichen Wettbewerbs sorgt für bestmögliche Befriedigung der Stakeholderinteressen: Das Ziel langfristiger Gewinnmaximierung kann nur der Unternehmer erreichen, der den Interessen der
Stakeholder weit entgegenkommt. Wer seinen Kunden alte Brötchen liefert, wer seine Kreditgeber hintergeht, wer seine Mitarbeiter respektlos behandelt, verfehlt das langfristige Gewinnmaximum, weil seine Vertragspartner sein Verhalten mit dem Abbruch der Geschäftsbeziehung sanktionieren" (Wöhe 2016, S. 10, im Original teilweise hervorgehoben). Wir finden eine weitergefasste Harmoniehypothese aber beispielsweise auch in dem Argument des Shareholder-Value-Ansatzes, dass die Shareholderorientierung zugleich die Interessen der Stakeholder berücksichtigt (Sundaram und Inkpen 2004). Ähnlich ist die Argumentation von Albach, der seine These, Unternehmensethik in der BWL sei verzichtbar, damit begründet, dass das Wirtschaftlichkeitsprinzip selbst ethisch gerechtfertigt sei (Albach 2005, S. 811 f.; kritisch Thielemann und Weibler 2007, S. 183-187). Ferner spielt sie in der Business Case-Vorstellung, dass sich verantwortliches Handeln von Unternehmen für Unternehmen bzw. ihre Anteilseigner lohnt (Carroll und Shabana 2010) eine Rolle. Hirschman (1986, S. 106-175) macht kritisch deutlich, dass solche und ähnliche Harmoniehypothesen eine lange Tradition haben.

Nach hier vertretener Auffassung ist diese Harmoniehypothese auch in einer weitergefassten Formulierung nicht empirisch bewährt. Diese weitergefasste Harmoniehypothese lautet, dass Handeln nach dem Prinzip, den (subjektiv erwarteten) eigenen Vorteil zu verfolgen, zur Berücksichtigung gesellschaftlicher Ziele führt, weil Wirtschaftssubjekte aus Eigeninteresse inr Handeln an den Interessen anderer orientieren. Beispielsweise bieten sie nachhaltige Güter an, weil diese im Interesse anderer sind. Wirtschaftssubjekte orientieren sich an den Interessen anderer Wirtschaftssubjekte, um diese zur Zustimmung zum Tausch zu motivieren (Buchanan und Vanberg 1991, S. 181 f.; Vanberg 2007, S. 202-207; Vanberg 2011, S. 3-7). Die folgende Abbildung 3 verdeutlicht diese weitgefasste Harmoniehypothese als Teil einer Ziel-Mittel-Analyse.

Diese Hypothese über den Marktmechanismus wird nach hier vertretener Auffassung dadurch eingeschränkt, dass häufig die betroffenen Wirtschaftssubjekte (typischerweise zukünftige Generationen) an dem Tauschakt nicht beteiligt sind, also externe Effekte vorliegen (Nutzinger 1992, S. 53). Selbst wenn die betroffenen Wirtschaftssubjekte beteiligt sind, spricht gegen die weitergefasste Harmoniehypothese die Kernhypothese der Resource-Dependence-Theorie. Danach nehmen Wirtschaftssubjekte die Interessen anderer Wirtschaftssubjekte durchaus in den Blick. Allerdings werden nicht die Interessen sämtlicher Wirtschaftssubjekte automatisch berücksichtigt. Vielmehr beachten Wirtschaftssubjekte die Interessen mächtiger Ressourcengeber 
Marktziel (Wert): Berücksichtigung Interessen anderer aus Eigeninteresse

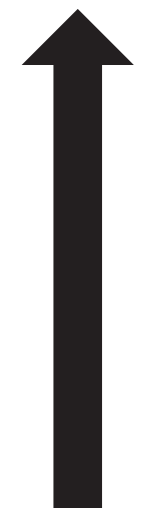

Zielerreichung durch Mittel:

Markthypothese: Wirtschaftssubjekte orientieren sich aus Eigeninteresse an den Interessen anderer, um diese zur Zustimmung zum Tausch zu motivieren

Mittel: Marktbedingungen: Zulässigkeit des Handelns im Eigeninteresse

Abbildung 3. Weitgefasste Harmoniehypothese als Teil einer Ziel-Mittel-Analyse

Quelle: Eigene Darstellung

(Pfeffer und Salancik 2003, S. 43-52, 257-262; allgemein zur Relevanz von Macht auf Märkten Fligstein 2011, S. 39-48; Aspers und Beckert 2017, S. 221 f.). Soweit davon auszugehen ist, dass Ressourcen absolut knapp und unterschiedlich verteilt sind und daraus Ressourcenmacht resultiert, werden ressourcenmächtige Wirtschaftssubjekte die Interessen ressourcenabhängiger Wirtschaftssubjekte nicht beachten, weil sie auf deren Bereitschaft zum Tausch nicht angewiesen sind. Umgekehrt reicht bei ressourcenabhängigen Wirtschaftssubjekten die Zustimmung zum Tausch allein nicht aus, um deren Interessen zur Geltung zu bringen (Thielemann 2008, S. 217-220; Hoßfeld und Schmiel 2015). Außerdem sind manche Wirtschaftssubjekte faktisch aufgrund ihrer geringen Ressourcenausstattung nicht in der Lage, einen Tausch abzulehnen. Ihnen fehlt die positive Freiheit (Berlin 2002a, S. 30-54; 2002b, S. 166-217) zu handeln, weil ihnen die notwendige Ressourcenausstattung fehlt (Sen 1988; van Aaken 2012, S. 86-90).

Die Hypothese, auf Märkten würden individuelle und gesellschaftliche Ziele „automatisch“ übereinstimmen, ist damit weder in Form der neoklassischen Harmoniehypothese noch in Form einer weitergefasste Harmoniehypothese vorläufig empirisch bewährt (siehe zur generellen Kritik der Harmoniehypothese Ulrich 2008, S. 175-215 und bereits Hirschman 1986; hierzu Aspers und Beckert 2017, S. 220 f.). Märkte erfordern Regelungen, sie sind soziale Arrangements (Vanberg 2001, S. 9225-9226; 2005, S. 37-41; Fligstein 2011, S. 39-56; Aspers und Beckert 2017). Die Ausgestaltung und Absicherung dieser Regelungen ist keineswegs trivial. So ist zu diskutieren, welche Interessen welcher Akteure aus ethischer Sicht berücksichtigt werden sollten. Ebenso ist zu untersuchen, wie abgesichert werden kann, dass Akteure diese Interessen anderer auch tatsächlich berücksichtigen. Es ist zu erwarten, dass es über Art und Umfang solcher zu berücksichtigender Interessen ebenso unterschiedliche Auffassungen geben wird wie zu der Frage, welche Regelungen Eigeninteressen und $z u$ berücksichtigende Interessen anderer Akteure adäquat koordinieren. Aus kritisch-rationaler Sicht lassen sich zu berücksichtigende Interessen nicht letztbegründen. Ebenso wenig können gesetzliche Regelungen entwickelt werden, die diese Koordination perfekt leisten. Vielmehr sind Rechtsordnungen immer unvollständig. Zum einen, weil Wissen unvollständig und zum anderen, weil es nicht möglich ist, alle zukünftigen Sachverhalte zu berücksichtigen. Diese Unvollständigkeit des Rechts wird außerdem dadurch verschärft, dass nationale Gesetze auf globalen Märkten nur eine begrenzte Reichweite haben (Burkatzki und Löhr 2008, S. 14-16). Diese Unvollständigkeit des Rechts wird unterschätzt, wenn davon ausgegangen wird, dass die Aufgabe des demokratisch legitimierten Gesetzgebers darin besteht, „mit Vorschriften zum Schutz der Umwelt und zum Schutz der Schwachen den ökologischen und sozialen Interessen der Stakeholder gerecht zu werden“" (Wöhe 2016, S. 10)

Es fehlt also an vorläufig bewährten empirischen Hypothesen, dass Handeln, das ausschließlich die eigene Vorteilhaftigkeit in den Blick nimmt, auch wenn es bestehende Gesetze befolgt, zu Pareto-Effizienz führt oder die Interessen anderer ausreichend in den Blick nimmt. Damit ist nicht begründet, dass die BWL die Interessen anderer Akteure in unternehmenspolitischen Ziel-Mittel-Analysen nicht explizit zu berücksichtigen braucht. Es reicht weder aus, dass die BWL in unternehmenspolitischen Ziel-Mittel-Analysen die Interessen anderer Akteure nur insoweit berücksichtigt, wie 
es aus Unternehmenssicht (bzw. aus Sicht der Unternehmenseigner) vorteilhaft ist noch genügt es, entsprechende gesetzliche Regelungen zu befolgen. Auf der Objektebene (wie sollten Unternehmen handeln?) finden wir ähnliche Ergebnisse, allerdings zum Teil mit unterschiedlicher Begründung (Donaldson und Preston 1995, S. 81-88; Freeman und Phillips 2002, S. 340; Thielemann 2008, S. 225; Hoßfeld und Schmiel 2015).

Abschließend lässt sich ergänzend gegen beide Ziel-Mittel-Aussagen, die Harmoniehypothesen zugrunde legen, einwenden, dass das jeweilige Ziel „Effizienz" einerseits bzw. „Berücksichtigung Interessen anderer aus Eigeninteresse" aus kritisch-rationaler Sicht nicht die Mittel heiligt. Vielmehr sind diese selbst (in unserem Beispiel der Vorschlag, die Investition mit dem höchsten Erwartungswert des Kapitalwerts zu wählen oder legale aggressive Steuergestaltung zu betreiben) aus ethischer und erfahrungswissenschaftlicher Perspektive kritisch zu reflektieren. Im Hinblick auf die oben genannten Beispiele wäre zu untersuchen, ob der Kapitalwert vor dem Hintergrund der zugrunde liegenden Prämisse eines vollkommenen Kapitalmarkts ein geeignetes Instrument zur Zielerreichung ist (Biondi und Marzo 2011). Des Weiteren ist zu untersuchen, ob Mittel aufgrund ihrer Nebenwirkungen mit anderen Zielen oder Mitteln in Konflikt treten (Albert 1985, S. 80-87, 218-229). Im Hinblick auf das Mittel aggressive Steuergestaltung wären beispielsweise die (fehlende) Vereinbarkeit mit der Finanzierung von Staatsausgaben zu untersuchen.

Das Ergebnis dieses Kapitels lautet damit: Es gibt keinen Grund dafür, die Interessen anderer Akteure in unternehmenspolitischen Ziel-Mittel-Analysen nicht explizit zu berücksichtigen. Nach hier vertretener Auffassung sollten deshalb die Interessen anderer Akteure in unternehmenspolitischen Ziel-Mittel-Analysen berücksichtigt werden. Damit stellt sich für die BWL als Wissenschaft die Frage, wie die Interessen anderer Akteure im Rahmen unternehmenspolitischer Ziel-Mittel-Analysen zu thematisieren sind. Diese Frage ist Gegenstand des Kapitels 5.

\section{Wie sollten unternehmenspolitische Ziel- Mittel-Analysen die Interessen anderer Akteure thematisieren?}

Dieser Beitrag argumentiert, dass die BWL in unternehmenspolitischen Ziel-Mittel-Analysen die Interessen anderer Akteure berücksichtigen sollte. Impliziert dies, dass Unternehmen unmittelbar die Interessen anderer Akteure, also unmittelbar das Gemeinwohl anstreben sollten? Nach hier vertretener Auffassung ist das nicht der Fall, weil eine solche Forderung dem hier zugrunde gelegten Marktverständnis widersprechen würde. Dieser Beitrag versteht Märkte als Institutionen, mit denen Interessen koordiniert werden (Vanberg 2005, S. 37 f.; Fligstein 2011, S. 42; Aspers und Beckert 2017). Aspers und Beckert (2017) unterscheiden in Anlehnung an Polanyi (1957) Redistribution, Reziprozität und Märkte als Mechanismen für die Produktion und Verteilung von Gütern. Redistribution ist durch die Verteilung von Gütern durch eine zentrale Instanz nach dem Kriterium der Bedürftigkeit charakterisiert. Reziprozität ist dagegen durch die Verteilung von Gütern mit der Verpflichtung zu einer späteren Erwiderung gekennzeichnet. In Abgrenzung dazu ist für Märkte der freiwillige Tausch konstitutiv, der durch das Erzielen wechselseitiger Nutzenvorteile motiviert ist. Nach diesem Verständnis des Koordinationsinstruments "Markt“ können Akteure ihre eigenen Interessen verfolgen, denn ansonsten wäre es nicht möglich, dass sie ihre Nutzenvorteile verfolgen. Deshalb würde es diesem Verständnis des Koordinationsinstruments „Markt“ widersprechen, wenn einzelne Akteure überhaupt nicht mehr an dem eigenen Vorteil orientiert handeln dürften, sondern immer unmittelbar die Interessen anderer Akteure anstreben sollten. Akteure sind nach diesem Verständnis also nicht dazu verpflichtet, uneingeschränkt unmittelbar die Interessen anderer Akteure anzustreben, sondern sie können sich grundsätzlich an ihren eigenen Zielen orientieren (Vanberg 2007, S. 202-207).

Vor dem Hintergrund der in Kapitel 4 erarbeiteten Ergebnisse wäre es jedoch nicht begründet, dass Akteure uneingeschränkt - also ohne Restriktionen ihre eigenen Interessen verfolgen können. So ist die Hypothese, dass Handeln, das ausschließlich die eigene Vorteilhaftigkeit in den Blick nimmt und bestehende Gesetze befolgt, zugleich gesellschaftlich vorteilhaft ist (also zu Pareto-Effizienz führt oder die legitimen Interessen anderer Akteure ausreichend in den Blick nimmt) nicht empirisch bewährt. Wenn aber der gesellschaftliche Wert von Märkten darin bestehen soll, dass Akteure im Eigeninteresse handeln können und dabei verstanden als Restriktionen - legitime Interessen anderer wahren sollen, reichen Analysen wie die einführend genannten Ziel-Mittel-Aussagen Wenn Unternehmen den Shareholder Value maximieren wollen, dann ist es bei Risikoneutralität ein geeignetes Mittel, die Investition mit dem höchsten Erwartungswert des Kapitalwertes zu wählen oder Wenn Unternehmen Gewinne maximieren wollen, dann ist legale aggressive Steuergestaltung ein geeignetes Mittel, sofern die Risiken abschätzbar und kein Reputationsschaden zu erwarten ist nicht aus. Vielmehr wären neben der Verfolgung des eigenen Vorteils auch legitime Interessen anderer Akteure zu 
berücksichtigen. Im Folgenden soll kurz beleuchtet werden, welche Fragen solche Analysen aufwerfen.

In Kapitel 4 wurde bereits deutlich, dass Wirtschaftssubjekte unter genuiner Unsicherheit nicht objektiv-rational, sondern nur subjektiv-rational handeln können. Akteure können nur vor dem Hintergrund ihres subjektiven Wissens ihre subjektiven Ziele verfolgen (Beckert 1996, S. 817-823; Vanberg 2002, S. 10-15). Das bedeutet aber, dass unter genuiner Unsicherheit keineswegs eindeutig ist, welches Handeln vorteilsorientiert ist. Dies ist ein wichtiger Unterschied zu vorteilsorientiertem Handeln von Unternehmen im Rahmen neoklassischer Theorie. Im Rahmen neoklassischer Markttheorie bedeutet perfekt rationales Handeln von Unternehmen, Zielgrößen wie den Ertragswert oder daraus abgeleitete Zielgrößen zu verfolgen (Biondi und Marzo 2011).

Unter genuiner Unsicherheit ist zu berücksichtigen, dass Zielgrößen wie der Ertragswert, der Kapitalwert, die Kapitalkosten oder daraus abgeleitete Größen ein neoklassisches Fundament haben (Schmidt und Spindler 1997, S. 523-527), sodass innen unrealistische und nicht realisierbare Antezedensbedingungen zugrunde liegen. Damit ist fraglich, ob es für Unternehmen subjektiv-rational ist, diese Zielgrößen zugrunde zu legen oder ob es aus Unternehmenssicht nicht vernünftiger wäre, andere Zielgrößen zu verfolgen (Biondi und Marzo 2011). Dass Unternehmen sich an neoklassischen Zielgrößen orientieren, könnte subjektiv-rational sein, wenn davon auszugehen ist, dass diese Zielgrößen als Deutungsmuster wirtschaftlich erfolgreicher Unternehmen verstanden werden (Meyer und Rowan 1977; Fligstein 2011, S. 44 f.). Die Frage nach subjektiv-rationalen Zielgrößen ist unter genuiner Unsicherheit nach hier vertretener Auffassung eine erste relevante Frage, die die BWL im Rahmen unternehmenspolitischer Ziel-Mittel-Analysen untersuchen sollte. Es wäre also bezogen auf die einführend genannten Aussagen zu überlegen, unter welchen Bedingungen Investitionsentscheidungen und auch andere Handlungen von Unternehmen vorteilhaft sind. Dabei stellt sich auch die Frage, ob die Vorteilhaftigkeit aus Sicht des Unternehmens mit der Vorteilhaftigkeit aus Sicht der Anteilseigner identisch ist. Die Beantwortung dieser Frage ist abhängig davon, ob Unternehmen im Sinne des nexus of contracts approach als legal fictions (Jensen und Meckling, 1976; Fama, 1980) oder ob zumindest große Unternehmen als korporative Akteure angesehen werden (Vanberg, 1992). Unabhängig davon, welche Zielgröße Unternehmen verwenden, ist zu bedenken, dass diese nicht automatisch legitime Interessen anderer Akteure berücksichtigen. Dass zwischen individuellem Interesse und Interessen anderer Akteure keine automatische Harmonie besteht, zeigt sich damit auch in betriebswirtschaftlichen Gewinngrößen wie dem Ertragswert (oder daraus abgeleiteten Größen) und ebenso im Konzept des HGB-Gewinns oder IFRS-Gewinns. Sie alle berücksichtigen Interessen anderer Akteure in Form zukünftiger Auszahlungen oder in Form von Verbindlichkeiten oder Rückstellungen nur soweit, wie dies gesetzlich vorgesehen ist oder wie andere Akteure dies in Verträgen ausgehandelt haben. Damit ist aber nicht gewährleistet, dass sie Interessen anderer Akteure in dem Umfang berücksichtigen, in dem sie legitim sind (Schmiel 2012, S. 317 f.).

Eine zweite relevante Frage der BWL im Zusammenhang mit unternehmenspolitischen Ziel-Mittelanalysen ist die Frage nach der Konkretisierung legitimer Interessen anderer Akteure. Weil die Verfolgung des eigenen Vorteils und gesellschaftliche Werte bei allen Handlungen konfligieren können, ist es notwendig, dass die BWL dieses Spannungsfeld auf allen Handlungsfeldern (beispielsweise im Hinblick auf Finanzierungs-, Investitions-, Produktions-, Organisations- oder Personalentscheidungen) untersucht. Dabei ist davon auszugehen, dass in Abhängigkeit von dem jeweiligen Handlungsfeld die Interessen unterschiedlicher Akteure betroffen sind. Bezogen auf die zu Beginn eingeführten Thematiken müsste untersucht werden, welche legitimen Interessen anderer Akteure von Investitionsentscheidungen betroffen sein könnten. Ebenso wäre zu analysieren, ob von aggressiver Steuergestaltung legitime Interessen anderer berührt sein könnten. Vorschläge über legitime Interessen anderer Akteure werden beispielsweise von der Wirtschaftsethik unterbreitet. So erörtern Crane und Matten Interessen von Anteilseignern, Arbeitnehmern, Konsumenten, Konkurrenten und anderen Stakeholdern (Crane und Matten 2016, S. 229-536), die sie als legitim erachten. Nach kritisch-rationalem Wissenschaftsverständnis müssten solche Vorschläge durch Argumente gestützt werden können. Für die Legitimität konkretisierter Interessen spräche beispielsweise, dass deren Berücksichtigung auf allgemeine Werte zurückgeführt werden kann, die ihrerseits begründet sind. Gegen die Legitimität von Interessen könnte sprechen, wenn deren Berücksichtigung anderen begründeten Werten widerspricht (Albert 1985, S. 71-87, 199-229; Beauchamp 2003). Beispielsweise konkretisiert Witt legitime Interessen im Hinblick auf aggressive Steuergestaltung. Weil aggressive Steuergestaltung andere Bürger in Form von Steuerausfällen schädigt und es nach einer allgemeineren moralischen Regel falsch ist, Personen zu schädigen, ist seiner Auffassung nach aggressive Steuergestaltung moralisch verwerflich (Witt 2017, S. 242246). Aus kritisch-rationaler Sicht könnte nun sowohl die allgemeine moralische Regel, dass es falsch ist, Personen zu schädigen als auch die Überlegung, ob 
andere Bürger durch Steuerausfälle geschädigt werden, kritisch diskutiert werden. Auch van Aaken greift bei der Konkretisierung legitimer Interessen anderer Akteure auf allgemeine Werte zurück. So konkretisiert er legitime Interessen von Vertragspartnern von Unternehmen. Gemäß diesen Überlegungen sind Verträge nur legitim, wenn beide Vertragspartner über Ressourcen verfügen, um einen Vertrag abzulehnen. Ist dies nicht der Fall, müssten Unternehmen Verträge so gestalten wie Vertragspartner, die über entsprechende Ressourcen verfügen, dies tun würden. Um die Legitimität dieser Interessen zu begründen, führt van Aaken als Argument den allgemeineren Wert positiver Freiheit an (van Aaken 2012). Und auch hier könnte aus kritisch-rationaler Sicht sowohl hinterfragt werden, ob positive Freiheit ein Wert der Marktwirtschaftsordnung darstellen sollte. Ebenso könnte kritisch diskutiert werden, ob die vorgelegte Konkretisierung positiver Freiheit geeignet ist.

Wie in Kapitel 3 deutlich wurde, haben Ziel-MittelAnalysen nicht nur eine ethische, sondern auch eine erfahrungswissenschaftliche Dimension. Danach ist es auch notwendig, diese auf ihre Realisierbarkeit hin zu untersuchen und verschiedene alternative Konkretisierungen auf ihre Realisierbarkeit hin miteinander zu vergleichen. Verbunden mit dem Realisierbarkeitspostulat ist zum einen die Frage, ob Unternehmen die für bestimmte Handlungsfelder konkretisierten Interessen anderer Akteure in einer Marktwirtschaft überhaupt beachten können. Fraglich ist beispielsweise, ob Unternehmen, die (bestimmte) legitime Interessen anderer Akteure berücksichtigen, dadurch ihre Existenz gefährden. Hieran schließt sich auch die Frage an, ob es Argumente dafür gibt, eine solche Nebenwirkung bei der Berücksichtigung der Interessen anderer Akteure in Kauf zu nehmen. Die erfahrungswissenschaftliche Dimension beschränkt sich nicht auf die Frage, ob Unternehmen konkretisierte legitime Interessen anderer berücksichtigen können. Ebenso stellt sich die Frage, ob Unternehmen gesellschaftliche Werte berücksichtigen wollen. Wenn gemäß der Kernhypothese des Resource-Dependence-Ansatzes Unternehmen nicht die (gemäß gesellschaftlichen Werten legitimen) Interessen anderer Wirtschaftssubjekte wahren, sondern nur die Interessen mächtiger Ressourcengeber erfüllen wollen (Pfeffer und Salancik 2003, S. 43-52, 257-262), ist zu erwarten, dass Unternehmen die Interessen ressourcenschwacher Akteure nicht berücksichtigen werden.

Sowohl der Einwand, dass Unternehmen legitime Interessen nicht berücksichtigen können als auch der Einwand, dass sie es nicht wollen, zeigen die Notwendigkeit auf, nicht nur moralische Regeln zu formulieren, sondern diese durch gesetzliche oder private Regulierung (beispielsweise CSR-Standards) abzusichern.
Beispielsweise wäre zu untersuchen, ob Selbstverpflichtungen durch CSR-Standards ein geeignetes Instrument zur Absicherung sein könnte, wenn von so genannten "Watchdogs", also beispielsweise von Medien oder NGOs, die Einhaltung dieser Standards kontrolliert wird. Diese könnten dazu führen, dass sich die Nichtberücksichtigung legitimer Interessen anderer Akteure über Reputationsverluste gewinnmindernd auswirkt (Hoßfeld und Schmiel 2015). Allerdings ist fraglich, ob CSR-Standards geeignete Instrumente sind, wenn man berücksichtigt, dass Unternehmen diese zum „Greenwashing“ nutzen könnten (Gröneweg und Matiaske 2012). Außerdem wäre auch zu untersuchen, wie "Watchdogs" handeln und ob dieses Handeln aus ethischer Sicht wünschenswert ist. Um die Frage nach der geeigneten Form der Absicherung beantworten zu können, sind damit auch erfahrungswissenschaftliche Hypothesen über die Wirkung solcher Absicherungsinstrumente erforderlich. Wenn der gesellschaftliche Wert von Märkten darin bestehen soll, dass Akteure im Eigeninteresse handeln können und dabei legitime Interessen anderer Akteure wahren sollen, dann sind also als Mittel Marktbedingungen erforderlich, die dies ermöglichen. Hierzu gehören gesetzliche und außergesetzliche Regeln, die es Unternehmen ermöglichen, den eigenen Vorteil zu verfolgen. Hierzu gehören auch - verstanden als Restriktionen - gesetzliche und außergesetzliche Regeln, die Unternehmen zur Beachtung konkretisierter legitimer Interessen anderer Akteure verpflichten und die damit die Möglichkeit, den eigenen Vorteil zu verfolgen, einschränken. Die folgende Abbildung 4 fasst diese Überlegungen zusammen.

\section{Fazit}

In der Standard-Lehrbuchliteratur wird auf einer methodologischen Ebene die Auffassung vertreten, die BWL bräuchte in unternehmenspolitischen Analysen die Interessen anderer Akteure nicht explizit zu berücksichtigen (Kapitel 2). Der vorliegende Beitrag hinterfragt dies und untersucht, ob sich die BWL bei unternehmenspolitischen Ziel-Mittel-Analysen auf die Vorteilhaftigkeit aus Sicht des Unternehmens bzw. der Anteilseigner beschränken kann oder ob sie explizit auch Interessen anderer Akteure berücksichtigen soll. Von den bisher vorliegenden methodologischen Erörterungen grenzt sich der Beitrag dadurch ab, dass er explizit eine kritisch-rationale Perspektive im Sinne von Albert (1985; 1999) und Gadenne (2006) einnimmt.

Aus kritisch-rationaler Sicht hat die Analyse von Ziel-Mittel-Aussagen zunächst eine ethische Dimension. Im Rahmen dieser ethischen Dimension sind Ziele und Mittel als alternative Vorschläge zu anderen Zielen und 
Marktziel (Wert): Berücksichtigung Interessen anderer aus Eigeninteresse

unter Wahrung legitimer Interessen anderer Akteure

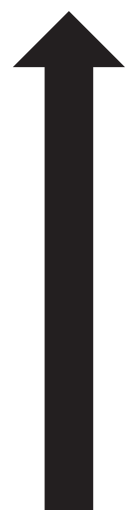

Zielerreichung durch Mittel:

Markthypothese: Wirtschaftssubjekte orientieren sich aus Eigeninteresse an den Interessen ressourcenmächtiger Akteure

Hypothesen darüber, wie abgesichert werden kann, dass Unternehmen legitime Interessen anderer Akteure wahren

Mittel: Marktbedingungen: gesetzliche/außergesetzliche Regeln, die grundsätzlich vorteilsorientiertes Handeln von Unternehmen ermöglichen. Gesetzliche/außergesetzliche Regeln, die absichern, dass konkretisierte legitime Interessen anderer Akteure gewahrt werden

Abbildung 4. Unternehmenspolitische Ziel-Mittel-Analysen in der BWL

Quelle: Eigene Darstellung

Mittel zu verstehen. Ziele sind durch Rückgriff auf allgemeinere Ziele oder Werte ethisch zu reflektieren und zur kritischen Diskussion zu stellen. Darüber hinaus hat die Analyse von Ziel-Mittel-Aussagen eine erfahrungswissenschaftliche Dimension. Aus dem Prinzip „Sollen impliziert Können“ (Albert 1985, S. 98) resultiert, dass Ziele realisierbar sein sollen. Realisierbarkeit erfordert, dass die Bedingungen, die gemäß erfahrungswissenschaftlicher Hypothesen für die Zielerreichung notwendig sind, auch geschaffen werden können. Eine kritisch-rationale Methodologie fordert außerdem, dass Antezedensbedingungen und Hypothesen vorläufig empirisch bewährt sind. Des Weiteren sind Mittel dahingehend zu untersuchen, ob sie zur Zielerreichung geeignet sind und ob sie gegebenenfalls Nebenwirkungen entfalten, die mit anderen Zielen oder Mitteln in Konflikt treten. Für die Analyse unternehmenspolitischer Ziel-Mittel-Aussagen folgt hieraus, dass diese nur dann die explizite Berücksichtigung der Interessen anderer Akteure ausblenden können, wenn dies auf allgemeinere Ziele zurückgeführt werden kann, die ethisch begründet und realisierbar sind (Kapitel 3).

Dass die BWL in unternehmenspolitischen ZielMittel-Analysen die Interessen anderer Akteure nicht explizit in den Blick zu nehmen braucht, wird implizit oder explizit mit der Harmonie zwischen Unternehmenszielen und Interessen anderer Akteure begründet. Dieser Beitrag wendet jedoch ein, dass die in der BWL vertretenen Harmoniehypothesen empirisch nicht bewährt sind. Gegen die neoklassisch formulierte Harmoniehypothese, dass rationales Handeln von Wirtschaftssubjekten und den Bedingungen eines allgemeinen
Konkurrenzmarktgleichgewichts zu Pareto-Effizienz führt, wird hier eingewandt, dass die Antezedensbedingungen vollkommener Märkte nicht realisiert und auch nicht realisierbar sind und sie damit die kritisch-rationale Anforderung der Realisierbarkeit von Umweltbedingungen nicht erfüllt. Gegen eine weitergefasste Harmoniehypothese, nach der Wirtschaftssubjekte aus Eigeninteresse die Interessen anderer Akteure berücksichtigen, spricht die Kernhypothese der Resource-Dependence-Theorie. Danach nehmen Wirtschaftssubjekte die Interessen mächtiger Ressourcengeber in den Blick (Pfeffer und Salancik 2003, S. 43-52, 257-262). Diese können sicher mit legitimen Interessen übereinstimmen, ebenso ist aber denkbar, dass sie hiervon abweichen. Damit ist nicht begründet, dass die Interessen anderer Akteure in unternehmenspolitischen Ziel-Mittel-Analysen nicht berücksichtigt werden brauchen. Vielmehr sollten sie nach hier vertretener Auffassung explizit berücksichtigt werden.

Nach hier vertretenem Marktverständnis können Akteure ihre eigenen Interessen verfolgen, allerdings können sie dies aufgrund der fehlenden empirischen Bewährung der Harmoniehypothesen nicht uneingeschränkt. Vielmehr sollte der gesellschaftliche Wert von Märkten darin bestehen, dass Akteure im Eigeninteresse handeln können und dabei legitime Interessen anderer Akteure wahren sollen. Hierzu sind Marktbedingungen erforderlich, die dies ermöglichen. Dies erfordert die Konkretisierung legitimer Interesse anderer Akteure auf allen unternehmerischen Handlungsfeldern. Nach kritisch-rationalem Wissenschaftsverständnis müssten solche Konkretisierungen durch Argumente 
gestützt werden können. Ein Argument dafür wäre beispielsweise, dass konkretisierte legitime Interessen auf allgemeine Werte zurückgeführt werden können, die ihrerseits begründet sind. Ein Argument gegen die Legitimität von Interessen wäre beispielsweise, wenn deren Berücksichtigung anderen begründeten Werten widerspricht. Weiter wäre zu untersuchen, ob Unternehmen

\section{Literaturverzeichnis}

Aaken, Dominik van (2012), Individuelle Freiheit als Grundlage normativer Ökonomik, in: ZfB Special Issue, 82. Jg./Heft 6, S. 81-102.

Albach, Horst (2005), Betriebswirtschaftslehre ohne Unternehmensethik, in: ZfB, 75. Jg., S. 809-831.

Albert, Hans (1985), Treatise on Critical Reason, Princeton.

Albert, Hans (1999), Between Social Science, Religion and Politics: Essays in Critical Rationalism, Amsterdam/Atlanta.

Aspers, Patrick und Jens Beckert (2017), Märkte, in: Andrea Maurer (Hg.), Handbuch der Wirtschaftssoziologie, 2. Aufl., Wiesbaden, S. 215-240.

Beauchamp, Tom L. (2003), The Nature of Applied Ethics, in: Raymond G. Frey und Christopher $\mathrm{H}$. Wellman (Hg.), A Companion to Applied Ethics, Malden, S. 1-16.

Beckert, Jens (1996), What is Sociological about Economic Sociology? Uncertainty and the Embeddedness of Economic Action, in: Theory and Society, Vol. 25/No. 6, S. 803-840.

Berlin, I. (2002a), Introduction, in: Henry Hardy (Hg.), Liberty, Oxford, S. 3-54.

Berlin, I. (2002b), Two Concepts of Liberty, in: Henry Hardy (Hg.), Liberty, Oxford, S. 166-217.

Biondi, Yuri und Guiseppe Marzo (2011), Decision Making Using Behavioral Finance for Capital Budgeting, in: H. Kent Baker und Philip English (Hg.), Capital Budgeting Valuation: Financial Analysis for Today's Investment Decision, Hoboken, New Jersey, S. 421-444.

Buchanan, James M. und Viktor J. Vanberg (1991), The Market as a Creative Process, in: Economics and Philosophy, Vol. 7/No. 2, S. 167-186.

Burkatzki, Eckhard und Albert Löhr (2008), Wirtschaftskriminalität und Ethik - einführende Anmerkungen, in: Albert Löhr und Eckhard Burkatzki (Hg.), Wirtschaftskriminalität und Ethik, Mering, S. 11-21.

Carroll, Archie B und Kareem M. Shabana (2010), The Business Case for Corporate Social Responsibility: A Review of Concepts, Research and Practice, legitime Interessen anderer Akteure berücksichtigen können und/oder wollen. Sowohl der Einwand, dass Unternehmen legitime Interessen nicht berücksichtigen können als auch der Einwand, dass sie es nicht wollen, zeigt die Notwendigkeit auf, nicht nur moralische Regeln zu formulieren, sondern diese durch gesetzliche und/ oder private Regulierung abzusichern (Kapitel 5).

in: International Journal of Management Reviews, Vol. 12/No. 1, S. 85-105.

Crane, Andrew und Dirk Matten (2016), Business Ethics: Managing Corporate Citizenship and Sustainability in the Age of Globalization, 4th ed., New York.

Donaldson, Thomas und Lee E. Preston (1995), The Stakeholder Theory of the Corporation: Concepts, Evidence, and Implications, in: Academy of Management Review, Vol. 20/No. 1, S. 65-91.

Etzioni, Amitai (2015), Common Good, in: Michael T. Gibbons (Hg.), The Encyclopedia of Political Thought, Malden, S. 603-610.

Fama, Eugene F. (1980), Agency Problems and the Theory of the Firm, in: Journal of Political Economy, Vol. 88/No. 2, S. 288-307.

Fligstein, Neil (2011), Die Architektur der Märkte, Wiesbaden.

Freeman, R. Edward und Robert A. Phillips (2002), Stakeholder Theory: A Libertarian Defense, in: Business Ethics Quarterly, Vol. 12/No. 3, S. 331-349.

Gadenne, Volker (2006), Methodological Rules, Rationality, and Truth, in: Colin Cheyne (Hg.), Rationality and Reality: Conversations with Alan Musgrave, Dordrecht, S. 97-107.

Gaus, Gerald (2010), The Idea and Ideal of Capitalism, in: George G. Brenkert und Tom L. Beauchamp (Hg.), Business Ethics, Oxford, S. 73-95.

Grotherr, Siegfried (2015), Wie weit geht die gesellschaftsrechtliche Verpflichtung des Vorstands zur (aggressiven) internationalen Steuerplanung?, in: Die Unternehmensbesteuerung, 6. Jg., S. 360-372.

Gröneweg, Christine und Wenzel Matiaske (2012), Gullivers Fesseln - Corporate Social Responsibility als Normbildung, Bericht Nr. 25, Werkstatt für Organisation und Personalforschung e. V. Berlin.

Heitfeldt, Mark und Ute Schmiel (2017), Was spricht für das Besteuerungsziel "Gleichmäßigkeit der Besteuerung“? - zugleich ein Beitrag zum Problem der Begründung von Normen, in: Werner Nienhüser und Ute Schmiel (Hg.), Steuern und Gesellschaft, Marburg, S. 95-126. 
Hirschman, Albert O. (1986), Rival Views of Market Society and Other Recent Essays, New York.

Holstein, Lars (2003), Nachhaltigkeit und neoklassische Ökonomik, Marburg.

Hoßfeld, Heiko und Ute Schmiel (2015), Corporate Social Responsibility in der Marktwirtschaft - ein erfahrungswissenschaftlich begründetes Konzept, in: Zeitschrift für Wirtschafts- und Unternehmensethik, 16. Jg./Heft 3, S. 313-338.

Jensen, Michael C. und William H. Meckling (1976), Theory of the Firm: Managerial Behavior, Agency Costs and Ownership Structure, in: Journal of Financial Economics, Vol. 3/No. 4, S. 305-360.

Kleinewefers, Henner (2008), Einführung in die Wohlfahrtsökonomie, Stuttgart.

Köhler, Richard, Hans-Ulrich Küpper und Andreas Pfingsten (2007), Betriebswirtschaftslehre, in: Richard Köhler, Hans-Ulrich Küpper und Andreas Pfingsten (Hg.), Handwörterbuch der Betriebswirtschaft, Stuttgart, S. 134-160.

Mas-Colell, Andreu, Michael D. Whinston und Jerry R. Green (1995), Microeconomic Theory, New York.

Meyer, John. W. und Brian Rowan, (1977), Institutionalized Organizations: Formal Structure as Myth and Ceremony, in: American Journal of Sociology, Vol. 83/No. 2, S. 340-363.

Nutzinger, Hans G. (1992), Der Begriff Verantwortung aus ökonomischer und sozialethischer Sicht, in: Karl Homann (Hg.), Aktuelle Probleme der Wirtschaftsethik, Berlin, S. 43-67.

Opp, Karl-Dieter (2014), Methodologie der Sozialwissenschaften, 7. Aufl., Wiesbaden.

Pfeffer, Jeffrey und Gerald R. Salancik (2003), The External Control of Organizations: A Resource Dependence Perspective, Stanford.

Polanyi, Karl (1957), The Economy as an Instituted Process, in: Karl Polanyi, Conrad M. Arensberg und Harry W. Pearson (Hg.), Trade and Market in the Early Empires, Chicago.

Popper, Karl R. (1968), The Logic of Scientific Discovery, 2nd ed., London.

Richter, Rudolf und Eirik G. Furubotn (2005), Institutions and Economic Theory, 2nd ed., Ann Arbor.

Schanz, Günther (2009), Wissenschaftsprogramme der Betriebswirtschaftslehre, in: Franz X. Bea und Marcell Schweitzer (Hg.), Allgemeine Betriebswirtschaftslehre, Bd. 1, 10. Aufl., Stuttgart, S. 81-159.

Schmidt, Reinhard H. und Gerald Spindler (1997), Shareholder-Value zwischen Ökonomie und Recht, in: Heinz-Dieter Assmann (Hg.): Wirtschafts- und Medienrecht in der offenen Demokratie, Heidelberg, S. 515-555.
Schmiel, Ute (2012), Ist der Ertragswert eine mögliche finanzwirtschaftliche Zielgröße für nachhaltiges wirtschaftliches Handeln?, in: Die Betriebswirtschaft, Jg. 72/Heft 4, S. 309-323.

Schneider, Dieter (2001), Betriebswirtschaftslehre, Geschichte und Methoden der Wirtschaftswissenschaft, Bd. 4, München.

Schweitzer, Marcell (2009), Einleitung: Grundfragen, in:, Franz X. Bea und Marcell Schweitzer (Hg.), Allgemeine Betriebswirtschaftslehre, Bd. 1, 10. Aufl., Stuttgart, S. 1-80.

Sen, Amartya (1988), Freedom of Choice, in: European Economic Review, Vol. 32/No. 2-3, S. 269-294.

Shackle, George L.S. (1972), Epistemics and Economics, Cambridge.

Shubik, Martin (2007), Chapter 5 Accounting and its relationship to general equilibrium theory, in: Yuri Biondi, Arnaldo Canziani und Thierry Kirat (Hg.), The Firm as an Entity: Implications for Economics, Accounting and the Law, Abingdon, S. 73-81.

Smith, Adam (1966), An Inquiry Into the Nature and Causes of the Wealth of Nations: Volume 1, New York.

Sundaram, Anant K. und Andrew Inkpen (2004), The Corporate Objective Revisited, in: Organization Science, Vol. 15/No. 3, S. 350-363.

Thielemann, Ulrich (2008), Unternehmensverantwortung ethisch ernst genommen: The Case against the Business Case und die Idee verdienter Reputation, in: Ludger Heidbrink und Alfred Hirsch (Hg.), Verantwortung als marktwirtschaftliches Prinzip, Frankfurt a. M., S. 199-230.

Thielemann, Ulrich und Jürgen Weibler (2007), Betriebswirtschaftslehre ohne Unternehmensethik? Vom Scheitern einer Ethik ohne Moral, in: ZfB, 77. Jg., S. 179-194.

Ulrich, Peter (2008), Integrative Wirtschaftsethik: Grundlagen einer lebensdienlichen Ökonomie, 4. Aufl., Bern.

Vanberg, Viktor (1992), Organizations as Constitutional Systems, in: Constitutional Political Economy, Vol. 3/No. 2, S. 223-253.

Vanberg, Viktor (2001), Markets and the Law, in Neil J. Smelser und Paul B. Baltes (Hg.), International Encyclopedia of the Social and Behavioral Sciences, 14, Amsterdam, S. 9221-9227.

Vanberg, Viktor (2002), Rational Choice vs. Program-based Behavior, in: Rationality and Society, Vol. 14, S. 7-54.

Vanberg, Viktor (2004), The Rationality Postulate in Economics: Its Ambiguity, Its Deficiency and Its Evolutionary Alternative, in: Journal of Economic Methodology, Vol. 11/No. 1, S. 1-29. 
Vanberg, Viktor (2005), Market and State: The Perspective of Constitutional Political Economy, in: Journal of Institutional Economics, Vol. 1/No. 1, S. 23-49.

Vanberg, Viktor (2007), Corporate Social Responsibility and the "Game of Catallaxy": The Perspective of Constitutional Economics, in: Constitutional Political Economy, Vol. 18/No. 2, S. 199-222.

Vanberg, Viktor (2011), Liberal constitutionalism, constitutional liberalism and democracy, in: Constitutional Political Economy, Vol. 22/No. 1, S. 1-20.

Vanberg, Viktor (2014), Evolving Preferences and Welfare Economics: The Perspective of Constitutional Political Economy, in: Jahrbücher für Nationalökonomie und Statistik, Vol. 234/No. 2-3, Stuttgart, S. 328-349.
Wagner, Franz W. (2014), Wagner, Franz W. (2014): Der Homo Oeconomicus als Menschenbild des Steuerrechts, in: DStR 2014, 52. Jg. Heft 24, S. 1133-1143.

Weise, Peter, Wolfgang Brandes, Thomas Eger und Manfred Kraft (2005), Neue Mikroökonomie, 5. Aufl., Heidelberg.

Witt, Ulrich (2009), Novelty and the Bounds of Unknowledge in Economics, in: Journal of Economic Methodology, Vol. 16/No.4, S. 361-375.

Witt, Karsten (2017), Aggressive Steuergestaltung, in: Werner Nienhüser und Ute Schmiel (Hg.), Steuern und Gesellschaft, Marburg, S. 239-264.

Wöhe, Günter (2016), Einführung in die Allgemeine Betriebswirtschaftslehre, 26. Aufl., München. 